\title{
Connections between Some Concepts of Polynomial Trichotomy for Noninvertible Evolution Operators in Banach Spaces
}

\author{
Mihai-Gabriel Babuţia and Nicolae Marian Seimeanu \\ Department of Mathematics, Faculty of Mathematics and Computer Science, West University of Timişoara, \\ V. Pârvan Boulevard No. 4, 300223 Timişoara, Romania \\ Correspondence should be addressed to Mihai-Gabriel Babuţia; mbabutia@math.uvt.ro
}

Received 2 July 2015; Accepted 7 October 2015

Academic Editor: Toka Diagana

Copyright (C) 2015 M.-G. Babuţia and N. M. Seimeanu. This is an open access article distributed under the Creative Commons Attribution License, which permits unrestricted use, distribution, and reproduction in any medium, provided the original work is properly cited.

\begin{abstract}
The present paper treats three concepts of nonuniform polynomial trichotomies for noninvertible evolution operators acting on Banach spaces. The connections between these concepts are established through numerous examples and counterexamples for systems defined on the Banach space of square-summable sequences.
\end{abstract}

\section{Introduction}

In the theory of asymptotic behavior of first-order differential equations, one of the main problems is to decompose the state space into a direct sum of subspaces on which the solutions of the given system have prescribed behavior. One of these behaviors can be modelled by the notion of exponential dichotomy, in which the state space is decomposed into a direct sum of two subspaces (the stable and unstable subspace) such that on the stable subspace the norm of the solution tends to zero (exponentially, polynomially, or with the aid of a general function) and on the unstable subspace the norm of the solution tends to infinity (usually with the same type of growth rate-exponential, polynomial, etc.-as the stable one). The notion of exponential dichotomy has its origins from the work of Perron in 1930 [1]. This field has seen a rich development in the last decades, as it can be seen from [2-11].

Another behavior given by the above-mentioned problem is the decomposition of the state space into three subspaces: a stable subspace, an unstable subspace, and a central manifold. The behavior on the stable and unstable subspaces is dichotomic, and, in addition, the solution of the system must be bounded (or have a growth property). This behavior is known in the literature as the trichotomy property. The trichotomy property was first defined by Sacker and Sell in [12], and, later on, the study was widely spread and many results were obtained (see [13-18] and the references therein).
This paper extends the above-mentioned study of the property of trichotomy in the case in which the decay, expansion, and growth on the stable, unstable, and central manifold, respectively, are described by a polynomial behavior. We study three concepts of polynomial trichotomy (both uniform and nonuniform) defined in the general case of noninvertible evolution operators: polynomial trichotomy, strong polynomial trichotomy, and weak polynomial trichotomy. We establish the connections between the three concepts and, with the aid of the examples and counterexamples from Section 5, on one hand we point out the existence of systems which possess the above-defined properties, and, on the other hand, we delimit the behaviors presented in this paper.

\section{Supplementary Families of Projections}

Throughout this paper, we will consider the following framework:

(i) $l^{2}(\mathbb{N}, \mathbb{R})$ will be the Banach space of all real valued sequences $x=\left(x_{n}\right)_{n \geq 0}$ satisfying

$$
\sum_{n=0}^{\infty}\left|x_{n}\right|^{2}<\infty
$$

endowed with the norm $\|x\|_{2}=\left(\sum_{n=0}^{\infty}\left|x_{n}\right|^{2}\right)^{1 / 2}$. 
(ii) $X$ will be be a real or complex Banach space and $\mathscr{B}(X)$ will be the Banach space of all bounded linear operators on $X$.

(iii) The norms on $X$ and $\mathscr{B}(X)$ will be denoted by $\|\cdot\|$.

(iv) The identity operator on $X$ is denoted by $I$.

(v) $\Delta$ will be the set defined by $\Delta=\left\{(t, s) \in \mathbb{R}_{+}^{2}: t \geq s \geq\right.$ $0\}$.

Definition 1. A mapping $P: \mathbb{R}_{+} \rightarrow \mathscr{B}(X)$ is called a family of projections on $X$ if

$$
P(t) P(t)=P(t), \quad \forall t \geq 0 .
$$

Definition 2. A family of projections $P: \mathbb{R}_{+} \rightarrow \mathscr{B}(X)$ is called

(i) polynomially bounded if there exist $M \geq 1$ and $\gamma \geq 0$ such that

$$
\|P(t)\| \leq M(t+1)^{\gamma}, \quad \forall t \geq 0 ;
$$

(ii) bounded if there exists $M \geq 1$ such that

$$
\|P(t)\| \leq M, \quad \forall t \geq 0 .
$$

Definition 3. Three families of projections $P, Q, R: \mathbb{R}_{+} \rightarrow$ $\mathscr{B}(X)$ are called supplementary if for all $t \geq 0$ one has that

$$
P(t)+Q(t)+R(t)=I .
$$

In what follows, we present two leading examples of families of projections which will be used in Section 5 .

Example 4. Let $X=l^{2}(\mathbb{N}, \mathbb{R})$ and $p: \mathbb{R}_{+} \rightarrow \mathbb{R}_{+}$be a nondecreasing function. For every $t \geq 0$ we define $P_{1}(t)$ : $l^{2}(\mathbb{N}, \mathbb{R}) \rightarrow l^{2}(\mathbb{N}, \mathbb{R})$ by

$$
P_{1}(t) x=\left(y_{n}(t)\right)_{n \geq 0},
$$

where

$$
\begin{aligned}
y_{3 n}(t) & =x_{3 n}+p(t) \cdot x_{3 n+1}, \\
y_{3 n+1}(t) & =0 \\
y_{3 n+2}(t) & =0
\end{aligned}
$$

$$
n \in \mathbb{N} \text {. }
$$

Let $t \geq 0$. One can see that $P_{1}(t)$ is linear and if $x=\left(x_{n}\right)_{n \geq 0} \in$ $l^{2}(\mathbb{N}, \mathbb{R})$, we have that

$$
\begin{aligned}
\left\|P_{1}(t) x\right\|_{2}^{2} & =\sum_{n=0}^{\infty}\left|y_{3 n}(t)\right|^{2} \\
& \leq\left[\left(\sum_{n=0}^{\infty}\left|x_{n}\right|^{2}\right)^{1 / 2}+p(t)\left(\sum_{n=0}^{\infty}\left|x_{n}\right|^{2}\right)^{1 / 2}\right]^{2} \\
& =[(1+p(t))]^{2} \cdot\|x\|_{2}^{2}
\end{aligned}
$$

from where it follows that $P_{1}(t) \in \mathscr{B}\left(l^{2}(\mathbb{N}, \mathbb{R})\right)$ and $\left\|P_{1}(t)\right\| \leq$ $1+p(t)$.

Moreover, let $t \geq 0$ and $\tilde{x}=\left(\widetilde{x}_{n}\right)_{n \geq 0}$ given by

$$
\begin{aligned}
\tilde{x}_{3 n} & =\tilde{x}_{3 n+2}=0, \\
\tilde{x}_{3 n+1} & =\frac{1}{3 n+2},
\end{aligned}
$$

$n \in \mathbb{N}$.

From

$$
\begin{aligned}
\left\|P_{1}(t) \tilde{x}\right\|_{2} & =\left(\sum_{n=0}^{\infty}\left|y_{3 n}(t)\right|^{2}\right)^{1 / 2} \\
& =p(t) \cdot\left(\sum_{n=0}^{\infty} \frac{1}{(3 n+2)^{2}}\right)^{1 / 2}=p(t) \cdot\|\tilde{x}\|_{2}
\end{aligned}
$$

it follows that $\left\|P_{1}(t)\right\| \geq p(t)$. From here we get that

$$
\max \{1, p(t)\} \leq\left\|P_{1}(t)\right\| \leq 1+p(t) \quad \forall t \in \mathbb{R}_{+} .
$$

Moreover, for $(t, x) \in \mathbb{R}_{+} \times l^{2}(\mathbb{N}, \mathbb{R})$ we define the family of projections $Q_{1}: \mathbb{R}_{+} \rightarrow \mathscr{B}(X)$ by $Q_{1}(t) x=\left(z_{n}(t)\right)_{n \geq 0}$, where

$$
\begin{aligned}
z_{3 n}(t) & =-p(t) x_{3 n+1}, \\
z_{3 n+1}(t) & =x_{3 n+1}, \\
z_{3 n+2}(t) & =0
\end{aligned}
$$

$n \in \mathbb{N}, t \geq 0$.

Moreover, for $(t, s, x) \in \Delta \times l^{2}(\mathbb{N}, \mathbb{R})$ one can see that

$$
\begin{aligned}
\left\|Q_{1}(s) x\right\|_{2}^{2} & =\sum_{n=0}^{\infty}\left|z_{n}(s)\right|^{2} \\
& =\sum_{n=0}^{\infty}\left|z_{3 n}(s)\right|^{2}+\sum_{n=0}^{\infty}\left|z_{3 n+1}(s)\right|^{2} \\
& =\left(1+p(s)^{2}\right) \sum_{n=0}^{\infty}\left|x_{3 n+1}\right|^{2} \\
& \leq\left(1+p(t)^{2}\right) \sum_{n=0}^{\infty}\left|x_{3 n+1}\right|^{2}=\left\|Q_{1}(t) x\right\|_{2}^{2} ;
\end{aligned}
$$

hence

$$
\begin{aligned}
& \left\|Q_{1}(s) x\right\|_{2} \leq\left\|Q_{1}(t) x\right\|_{2}, \\
& \left\|Q_{1}(t) x\right\|_{2}=\sqrt{1+p(t)^{2}} \cdot\left(\sum_{n=0}^{\infty}\left|x_{3 n+1}\right|^{2}\right)^{1 / 2} .
\end{aligned}
$$
where

Finally, define $R_{1}: \mathbb{R}_{+} \rightarrow \mathscr{B}(X)$ by $R_{1}(t)=\left(w_{n}(t)\right)_{n \geq 0}$,

$$
\begin{aligned}
w_{3 n}(t) & =0, \\
w_{3 n+1}(t) & =0, \\
w_{3 n+2}(t) & =x_{3 n+2},
\end{aligned}
$$


We have that $R_{1}$ is bounded with

$$
\left\|R_{1}(t)\right\|=1, \quad \forall t \geq 0
$$

and moreover the families of projections $P_{1}, Q_{1}$, and $R_{1}$ are supplementary.

Example 5. Let $X=l^{2}(\mathbb{N}, \mathbb{R})$ and define $P_{2}, Q_{2}, R_{2}: \mathbb{R}_{+} \rightarrow$ $\mathscr{B}\left(l^{2}(\mathbb{N}, \mathbb{R})\right)$ by $P_{2}(t) x=\left(y_{n}(t)\right)_{n \geq 0}, Q_{2}(t) x=\left(z_{n}(t)\right)_{t \geq 0}$, and $R_{2}(t) x=\left(w_{n}(t)\right)_{n \geq 0}$, where, for $n \in \mathbb{N}$,

$$
\begin{aligned}
y_{4 n}(t) & =x_{4 n}, \\
y_{4 n+1}(t) & =0, \\
y_{4 n+2}(t) & =0, \\
y_{4 n+3}(t) & =0, \\
z_{4 n}(t) & =0, \\
z_{4 n+1}(t) & =x_{4 n+1}, \\
z_{4 n+2}(t) & =x_{4 n+2}, \\
z_{4 n+3}(t) & =0, \\
w_{4 n}(t) & =0, \\
w_{4 n+1}(t) & =0, \\
w_{4 n+2}(t) & =0, \\
w_{4 n+3}(t) & =x_{4 n+3} .
\end{aligned}
$$

We have that $P_{2}, Q_{2}$, and $R_{2}$ are three supplementary families of projections with

$$
\left\|P_{2}(t)\right\|=\left\|Q_{2}(t)\right\|=\left\|R_{2}(t)\right\|=1 \quad \forall t \geq 0 .
$$

\section{Evolution Operators}

Definition 6. A mapping $\Phi: \Delta \rightarrow \mathscr{B}(X)$ is called an evolution operator on $X$ if

$$
\begin{aligned}
& \left(e_{1}\right) \Phi(t, t)=I, \forall t \geq 0 ; \\
& \left(e_{2}\right) \Phi(t, s) \Phi\left(s, t_{0}\right)=\Phi\left(t, t_{0}\right), \forall(t, s),\left(s, t_{0}\right) \in \Delta .
\end{aligned}
$$

Definition 7. A family of projections $P: \mathbb{R}_{+} \rightarrow \mathscr{B}(X)$ is said to be invariant for the evolution operator $\Phi: \Delta \rightarrow \mathscr{B}(X)$ if

$$
\Phi(t, s) P(s)=P(t) \Phi(t, s) \quad \forall(t, s) \in \Delta .
$$

Given three supplementary families of projections $P, Q$, and $R$ which are invariant for a given evolution operator $\Phi$, we will name the quadruple $(\Phi, P, Q, R)$ a trichotomy quadruple.

Two important examples of trichotomy quadruples are given below, which will serve as a milestone in our examples and counterexamples.

Example 8. On $X=l^{2}(\mathbb{N}, \mathbb{R})$ consider the families of projections $P_{1}, Q_{1}$, and $R_{1}$ from Example 4. Consider $\varphi$ : $\mathbb{R}_{+} \rightarrow(0, \infty)$ and $\Phi_{1}: \Delta \rightarrow \mathscr{B}\left(l^{2}(\mathbb{N}, \mathbb{R})\right)$ given by

$$
\Phi_{1}(t, s)=\frac{\varphi(s)}{\varphi(t)} \cdot P_{1}(s)+\frac{\varphi(t)}{\varphi(s)} \cdot Q_{1}(t)+R_{1}(s)
$$

for all $(t, s) \in \Delta$.
Taking into account that for all $t, s \in \mathbb{R}_{+}$the following relations hold,

$$
\begin{gathered}
P_{1}(t) P_{1}(s)=P_{1}(s), \\
Q_{1}(t) Q_{1}(s)=Q_{1}(t),
\end{gathered}
$$

it follows that $\Phi_{1}$ is an evolution operator. It is easy to check that $P_{1}, Q_{1}$, and $R_{1}$ are invariant for $\Phi_{1}$; hence $\left(\Phi_{1}, P_{1}, Q_{1}, R_{1}\right)$ is a trichotomy quadruple. Moreover we have that

$$
\begin{aligned}
& \Phi_{1}(t, s) P_{1}(s)=\frac{\varphi(s)}{\varphi(t)} P_{1}(s), \\
& \Phi_{1}(t, s) Q_{1}(s)=\frac{\varphi(t)}{\varphi(s)} Q_{1}(t) \\
& \Phi_{1}(t, s) R_{1}(s)=R_{1}(s)
\end{aligned}
$$

for all $(t, s) \in \Delta$.

Example 9. On $X=l^{2}(\mathbb{N}, \mathbb{R})$ consider $P_{2}, Q_{2}$, and $R_{2}$ to be the families of projections defined in Example 5. For $\psi: \mathbb{R}_{+} \rightarrow$ $(0, \infty)$ define $\Phi_{2}: \Delta \rightarrow \mathscr{B}\left(l^{2}(\mathbb{N}, \mathbb{R})\right)$ by

$$
\Phi_{2}(t, s) x= \begin{cases}\left(y_{n}(t, s)\right)_{n \geq 0} & \text { if } t>s \\ x, & \text { if } t=s\end{cases}
$$

where

$$
\begin{aligned}
y_{4 n}(t, s) & =\frac{\psi(s)}{\psi(t)} x_{4 n}, \\
y_{4 n+1}(t, s) & =\frac{\psi(t)}{\psi(s)} x_{4 n+1}, \\
y_{4 n+2}(t, s) & =0 \\
y_{4 n+3}(t, s) & =x_{4 n+3},
\end{aligned}
$$

for all $(t, s, x) \in \Delta \times l^{2}(\mathbb{N}, \mathbb{R})$.

It is easy to see that $\left(\Phi_{2}, P_{2}, Q_{2}, R_{2}\right)$ is a trichotomy quadruple and for $(t, s, x) \in \Delta \times l^{2}(\mathbb{N}, \mathbb{R})$ one has that

$$
\begin{aligned}
\Phi_{2}(t, s) P_{2}(s) x & =\left(p_{n}(t, s)\right)_{n \geq 0}, \\
p_{4 n}(t, s) & =\frac{\psi(s)}{\psi(t)} x_{4 n}, \\
p_{4 n+1}(t, s) & =p_{4 n+2}(t, s)=p_{4 n+3}(t, s)=0 . \\
\Phi_{2}(t, s) Q_{2}(s) x & = \begin{cases}\left(q_{n}(t, s)\right)_{n \geq 0}, & t>s \\
\left(\rho_{n}(t, s)\right)_{n \geq 0}, & t=s\end{cases}
\end{aligned}
$$


which is given by

$$
\begin{aligned}
q_{4 n}(t, s) & =q_{4 n+3}(t, s)=0, \\
q_{4 n+1}(t, s) & =\frac{\psi(t)}{\psi(s)} x_{4 n+1}, \\
q_{4 n+2}(t, s) & =0, \\
\rho_{4 n}(t, s) & =\rho_{4 n+3}(t, s)=0, \\
\rho_{4 n+1}(t, s) & =x_{4 n+1}, \\
\rho_{4 n+2}(t, s) & =x_{4 n+2},
\end{aligned}
$$

and $\Phi_{2}(t, s) R_{2}(s) x=\left(r_{n}(t, s)\right)_{n \geq 0}$, where

$$
\begin{aligned}
r_{4 n}(t, s) & =r_{4 n+1}(t, s)=r_{4 n+2}(t, s)=0, \\
r_{4 n+3}(t, s) & =x_{4 n+3} .
\end{aligned}
$$

In what follows, we will present the main trichotomy concepts that will be studied in the present paper.

\section{Polynomial Trichotomies}

Definition 10. A trichotomy quadruple $(\Phi, P, Q, R)$ is said to be polynomially trichotomic (p.t.) if there exist $N \geq 1, \alpha>0$, and $\beta \geq 0$ such that

$$
\begin{aligned}
& \left(\mathrm{pt}_{1}\right)(t+1)^{\alpha}\|\Phi(t, s) P(s)\| \leq N(s+1)^{\alpha+\beta} ; \\
& \left(\mathrm{pt}_{2}\right)(t+1)^{\alpha} \leq N(t+1)^{\beta}(s+1)^{\alpha}\|\Phi(t, s) Q(s)\| ; \\
& \left(\mathrm{pt}_{3}\right)(s+1)^{\alpha}\|\Phi(t, s) R(s)\| \leq N(t+1)^{\alpha}(s+1)^{\beta} ; \\
& \left(\mathrm{pt}_{4}\right)(s+1)^{\alpha} \leq N(t+1)^{\alpha+\beta}\|\Phi(t, s) R(s)\|
\end{aligned}
$$

for all $(t, s) \in \Delta$.

If $\beta$ from the above definition is equal to 0 , then we say that $(\Phi, P, Q, R)$ is uniformly polynomially trichotomic (u.p.t.).

Remark 11. The following assertions hold:

(i) If a trichotomy quadruple $(\Phi, P, Q, R)$ is (p.t.) then $P$ and $R$ are polynomially bounded, and hence $Q$ is also polynomially bounded.

(ii) If a trichotomy quadruple $(\Phi, P, Q, R)$ is (u.p.t.) then $P$ and $R$ are bounded, and hence $Q$ is also bounded.

In other words, if $(\Phi, P, Q, R)$ is (p.t.) with constants $N, \alpha$, and $\beta$ then

$$
\max \{\|P(t)\|,\|Q(t)\|,\|R(t)\|\} \leq 3 N(t+1)^{\beta},
$$

$$
\forall t \geq 0 \text {. }
$$

Remark 12. If $(\Phi, P, Q, R)$ is (u.p.t.) then it is (p.t.). The converse is not generally true. Take, for example, the trichotomy quadruple $\left(\Phi_{1}, P_{1}, Q_{1}, R_{1}\right)$ from Example 8 with $p(t)=\varphi(t)=$ $t+1$. It is easy to check that $\left(\Phi_{1}, P_{1}, Q_{1}, R_{1}\right)$ is (p.t.), but it cannot be (u.p.t.), because $P$ is not bounded.
Definition 13. A trichotomy quadruple $(\Phi, P, Q, R)$ is said to be strongly polynomially trichotomic (s.p.t.) if there exist $N \geq$ $1, \alpha>0$, and $\beta \geq 0$ such that

$$
\begin{aligned}
& \left(\mathrm{spt}_{1}\right)(t+1)^{\alpha}\|\Phi(t, s) P(s) x\| \leq N(s+1)^{\alpha+\beta}\|P(s) x\| ; \\
& \left(\mathrm{spt}_{2}\right)(t+1)^{\alpha}\|Q(s) x\| \leq N(t+1)^{\beta}(s+1)^{\alpha}\|\Phi(t, s) Q(s) x\| ; \\
& \left(\mathrm{spt}_{3}\right)(s+1)^{\alpha}\|\Phi(t, s) R(s) x\| \leq N(t+1)^{\alpha}(s+1)^{\beta}\|R(s) x\| ; \\
& \left(\operatorname{spt}_{4}\right)(s+1)^{\alpha}\|R(s) x\| \leq N(t+1)^{\alpha+\beta}\|\Phi(t, s) R(s) x\| \\
& \text { for all }(t, s, x) \in \Delta \times X .
\end{aligned}
$$

If $\beta$ from the above definition is equal to 0 , then we say that $(\Phi, P, Q, R)$ is uniformly strongly polynomially trichotomic (u.s.p.t.).

Remark 14. If ( $\Phi, P, Q, R)$ is (u.s.p.t.) then it is (s.p.t.). The converse is not generally true, as shown in Example 1.

Remark 15. If $(\Phi, P, Q, R)$ is (s.p.t.) then the following condition holds:

$$
\text { Range } Q(s) \cap \operatorname{Ker} \Phi(t, s)=\{0\} \quad \forall(t, s) \in \Delta .
$$

In other words, for all $(t, s) \in \Delta$,

$$
\begin{array}{r}
x \in \operatorname{Range} Q(s), \\
\Phi(t, s) x=0 \Longrightarrow x=0 .
\end{array}
$$

Remark 16. Under the same assumption as in Remark 15, we also have that

$$
\text { Range } R(s) \cap \operatorname{Ker} \Phi(t, s)=\{0\} \quad \forall(t, s) \in \Delta .
$$

Definition 17. A trichotomy quadruple $(\Phi, P, Q, R)$ is said to be weakly polynomially trichotomic (w.p.t.) if there exist $N \geq$ $1, \alpha>0$, and $\beta \geq 0$ such that

$\left(\mathrm{wpt}_{1}\right)(t+1)^{\alpha}\|\Phi(t, s) P(s)\| \leq N(s+1)^{\alpha+\beta}\|P(s)\| ;$

$\left(\mathrm{wpt}_{2}\right)(t+1)^{\alpha}\|Q(s)\| \leq N(t+1)^{\beta}(s+1)^{\alpha}\|\Phi(t, s) Q(s)\|$;

$\left(\mathrm{wpt}_{3}\right)(s+1)^{\alpha}\|\Phi(t, s) R(s)\| \leq N(t+1)^{\alpha}(s+1)^{\beta}\|R(s)\|$;

$\left(\mathrm{wpt}_{4}\right)(s+1)^{\alpha}\|R(s)\| \leq N(t+1)^{\alpha+\beta}\|\Phi(t, s) R(s)\|$

for all $(t, s) \in \Delta$.

If $\beta=0$ then we say that $(\Phi, P, Q, R)$ is uniformly weakly polynomially trichotomic (u.w.p.t.).

Remark 18. If $(\Phi, P, Q, R)$ is (u.w.p.t.) then it is (w.p.t.). The converse is not generally true, as shown in Example 2.

In what follows we will study the connections between these three trichotomy concepts.

Remark 19. If a trichotomy quadruple $(\Phi, P, Q, R)$ is (s.p.t.) then it is also (w.p.t.). Moreover, if $(\Phi, P, Q, R)$ is (u.s.p.t.), then it is (u.w.p.t.).

Proposition 20. Let $(\Phi, P, Q, R)$ be a trichotomy quadruple. If $(\Phi, P, Q, R)$ is (p.t.) then it is also (w.p.t.). 
Proof. Let $N \geq 1, \alpha>0$, and $\beta \geq 0$ be given by Definition 10 . By Remark 11, we have that

$$
1 \leq \max \{\|P(t)\|,\|Q(t)\|,\|R(t)\|\} \leq 3 N(t+1)^{\beta}
$$

Let now $(t, s) \in \Delta$. From the estimations

$$
\begin{aligned}
(t & +1)^{\alpha}\|\Phi(t, s) P(s)\| \leq N(s+1)^{\alpha+\beta} \\
& \leq 3 N^{2}(s+1)^{\alpha+2 \beta}\|P(s)\| \\
(t & +1)^{\alpha}\|Q(s)\| \\
& \leq N(t+1)^{\beta}(s+1)^{\alpha}\|\Phi(t, s) Q(s)\| \cdot\|Q(s)\| \\
& \leq 3 N^{2}(s+1)^{\alpha}(t+1)^{2 \beta}\|\Phi(t, s) Q(s)\| \\
(s & +1)^{\alpha}\|\Phi(t, s) R(s)\| \leq N(t+1)^{\alpha}(s+1)^{\beta} \\
& \leq N(t+1)^{\alpha}(s+1)^{\beta}\|R(s)\| \\
& \leq 3 N^{2}(t+1)^{\alpha}(s+1)^{2 \beta}\|R(s)\|, \\
(s & +1)^{\alpha}\|R(s)\| \leq N(t+1)^{\alpha+\beta}\|\Phi(t, s) R(s)\| \cdot\|R(s)\| \\
& \leq 3 N^{2}(t+1)^{\alpha+\beta}(s+1)^{\beta}\|\Phi(t, s) R(s)\| \\
& \leq 3 N^{2}(t+1)^{\alpha+2 \beta}\|\Phi(t, s) R(s)\|,
\end{aligned}
$$

it follows that $(\Phi, P, Q, R)$ is (w.p.t.) with constants $3 N^{2} \geq 1$, $\alpha>0$, and $2 \beta \geq 0$.

Remark 21. From the proof of the above proposition, we can easily see that, by setting $\beta=0$, we obtain the implication (u.p.t.) $\Rightarrow$ (u.w.p.t.).

Other connections are given by the following.

Remark 22. (i) (s.p.t.) does not imply (p.t.) and (u.s.p.t.) does not imply (u.p.t.) as shown by Example 3 .

(ii) The concepts of (p.t.) and (w.p.t.) do not coincide, as we can see from Example 4.

(iii) (p.t.) does not imply (s.p.t.) and (u.p.t.) does not imply (u.s.p.t.), as shown by Example 5.

(iv) (w.p.t.) does not imply (s.p.t.) and (u.w.p.t.) does not imply (u.s.p.t.) as shown in Example 6.

Remark 23. The connection between the presented concepts is given by the following diagram:

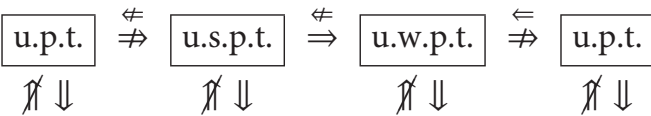

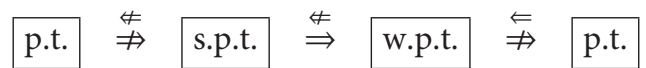

\section{Examples and Counterexamples}

Example 1 (trichotomy quadruple that is (s.p.t.) but not (u.s.p.t.)). Let $\left(\Phi_{1}, P_{1}, Q_{1}, R_{1}\right)$ be the trichotomy quadruple from Example 8 with $\varphi(t)=(t+1)^{3-\cos \ln (t+1)}$ and $p(t)=0$, $t \geq 0$. Then we have that

$$
\begin{aligned}
& (t+1)^{2}\left\|\Phi_{1}(t, s) P_{1}(s) x\right\|_{2} \leq(s+1)^{4}\left\|P_{1}(s) x\right\|_{2}, \\
& (t+1)^{2}\left\|Q_{1}(s) x\right\|_{2} \leq(s+1)^{4}\left\|\Phi_{1}(t, s) Q_{1}(s) x\right\|_{2}, \\
& \|\Phi(t, s) R(s) x\|_{2}=\|R(s) x\|_{2}
\end{aligned}
$$

for all $(t, s, x) \in \Delta \times X$; hence $\left(\Phi_{1}, P_{1}, Q_{1}, R_{1}\right)$ is (s.p.t.).

Assume, by a contradiction, that $(\Phi, P, Q, R)$ is (u.s.p.t.). Then there exist $N \geq 1$ and $\alpha>0$ such that for all $n \in \mathbb{N}$ and for $t_{n}=e^{2 n \pi}-1$ and $s_{n}=e^{2 n \pi-\pi / 2}-1$ we have, from $\left(\operatorname{spt}_{1}\right)$, that

$$
\frac{\left(e^{2 n \pi-\pi / 2}\right)^{4}}{e^{4 n \pi}} \leq N\left(\frac{e^{2 n \pi-\pi / 2}}{2^{2 n \pi}}\right)^{\alpha}
$$

which leads us to the contradiction

$$
e^{2 n \pi} \leq N e^{-\pi / 2}, \quad \forall n \in \mathbb{N}
$$

Example 2 (trichotomy quadruple that is (w.p.t.) but not (u.w.p.t.)). Let $\left(\Phi_{1}, P_{1}, Q_{1}, R_{1}\right)$ be as in Example 1. By Remark 19 we have that $\left(\Phi_{1}, P_{1}, Q_{1}, R_{1}\right)$ is (w.p.t.). The same contradiction is obtained as in Example 1, by assuming that $\left(\Phi_{1}, P_{1}, Q_{1}, R_{1}\right)$ is (u.w.p.t.).

Example 3 (trichotomy quadruple which is (s.p.t.) but fails to be (p.t.)). Let $\left(\Phi_{1}, P_{1}, Q_{1}, R_{1}\right)$ be the trichotomy quadruple from Example 8 with $p(t)=(t+1)^{t+1}$ and $\varphi(t)=t+1$.

From

$$
\begin{aligned}
& (t+1)\left\|\Phi_{1}(t, s) P_{1}(s) x\right\|_{2}=(s+1)\left\|P_{1}(s) x\right\|_{2}, \\
& (t+1)\left\|Q_{1}(s) x\right\|_{2} \leq \varphi(t)\left\|Q_{1}(t) x\right\|_{2} \\
& \quad=(s+1)\left\|\Phi_{1}(t, s) Q_{1}(s) x\right\|_{2}, \\
& (s+1)\|\Phi(t, s) R(s) x\|_{2} \leq(s+1)(t+1)\|R(s) x\|_{2}, \\
& (s+1)\|R(s) x\|_{2} \leq N(t+1)^{2}\|\Phi(t, s) R(s) x\|_{2}
\end{aligned}
$$

for all $(t, s, x) \in \Delta \times X$, we can see that $\left(\Phi_{1}, P_{1}, Q_{1}, R_{1}\right)$ is (u.s.p.t.) and hence (s.p.t.).

Assume, by a contradiction, that $\left(\Phi_{1}, P_{1}, Q_{1}, R_{1}\right)$ is (p.t.). Then, by Remark 11, we have that there exist $M \geq 1, \gamma \geq 0$, such that

$$
\left\|P_{1}(t)\right\| \leq M(t+1)^{\gamma} \quad \forall t \geq 0 .
$$

This leads us to the contradiction

$$
(t+1)^{t+1}=p(t) \leq\left\|P_{1}(t)\right\| \leq M(t+1)^{\gamma} \quad \forall t \geq 0 .
$$

It follows that $\left(\Phi_{1}, P_{1}, Q_{1}, R_{1}\right)$ is not (p.t.) and hence not (u.p.t.). 
Example 4 (trichotomy quadruple which is (w.p.t.) but not (p.t.)). Let $\left(\Phi_{1}, P_{1}, Q_{1}, R_{1}\right)$ the trichotomy quadruple from Example 3. By Remark 19, we have that $\left(\Phi_{1}, P_{1}, Q_{1}, R_{1}\right)$ is (u.w.p.t.) and hence (w.p.t.). But, by Example 3, it is not (p.t.) and hence not (u.p.t.).

Example 5 (trichotomy quadruple which is (p.t.) but fails to be (s.p.t.)). Let $\left(\Phi_{2}, P_{2}, Q_{2}, R_{2}\right)$ be the trichotomy quadruple from Example 9 with $\psi(t)=t+1$. First of all we will show that $\left(\Phi_{2}, P_{2}, Q_{2}, R_{2}\right)$ is (u.p.t.). Let $(t, s, x) \in \Delta \times l^{2}(\mathbb{N}, \mathbb{R})$. We have that

$$
\begin{gathered}
(t+1)^{2}\left\|\Phi_{2}(t, s) P_{2}(s) x\right\|_{2}^{2}=\sum_{n=0}^{\infty}\left|p_{n}(t, s)\right|^{2} \\
=(s+1)^{2} \sum_{n=0}^{\infty}\left|x_{4 n}\right|^{2}=(s+1)^{2}\left\|P_{2}(s) x\right\|_{2}^{2}
\end{gathered}
$$

hence

$$
(t+1)\left\|\Phi_{2}(t, s) P_{2}(s)\right\| \leq s+1
$$

If $t>s$, consider $x=\left(x_{n}\right)_{n \geq 0}$ given by

$$
x_{n}= \begin{cases}\frac{1}{n}, & n=4 k+1 \\ 0, & \text { otherwise }\end{cases}
$$

We have that $\|x\|_{2}=\left(\sum_{n=0}^{\infty}\left(1 /(4 n+1)^{2}\right)\right)^{1 / 2}$ and

$$
\begin{aligned}
(s+1)\left\|\Phi_{2}(t, s) Q_{2}(s) x\right\|_{2}=\left(\sum_{n=0}^{\infty}\left|q_{n}(t, s)\right|^{2}\right)^{1 / 2} \\
=(t+1)\|x\|_{2} ;
\end{aligned}
$$

hence

$$
t+1 \leq(s+1)\left\|\Phi_{2}(t, s) Q_{2}(s)\right\| .
$$

Having in mind that $\left\|\Phi_{2}(t, s) R(s) x\right\|_{2}=\|R(s) x\|_{2}$, it follows that $\left(\mathrm{spt}_{3}\right)$ and $\left(\mathrm{spt}_{4}\right)$ hold for $(t, s, x) \in \Delta \times X$ with $t>s$. The case in which $t=s$ obviously leads us to the above estimation, and so the conclusion follows.

In what follows, we will show that $\left(\Phi_{2}, P_{2}, Q_{2}, R_{2}\right)$ is not (s.p.t.), and hence it is not (u.s.p.t.). Assume by a contradiction that $\left(\Phi_{2}, P_{2}, Q_{2}, R_{2}\right)$ is (s.p.t.). We will disprove the result from Remark 15. Let $x=\left(x_{n}\right)_{n \geq 0}$ given by

$$
\begin{aligned}
& x_{4 n+2}=\frac{1}{4 n+2}, \\
& x_{4 n+3}=x_{4 n+1}=x_{4 n}=0 \\
& n \in \mathbb{N} .
\end{aligned}
$$

Obviously $x \in l^{2}(\mathbb{N}, \mathbb{R})$ and denote, for every $s \geq 0, Q_{2}(s) x=$ $\left(z_{n}(s)\right)_{n \geq 0}$, where

$$
\begin{aligned}
z_{4 n}(s) & =0 \\
z_{4 n+1}(s) & =x_{4 n+1}=0 \\
z_{4 n+2}(s) & =x_{4 n+2}=\frac{1}{4 n+2}, \\
z_{4 n+3}(s) & =0
\end{aligned}
$$

hence $\left(z_{n}(s)\right)_{n \geq 0}$ is a nonzero sequence.
Consider now $(t, s) \in \Delta$ with $t>s$. By denoting $\Phi_{2}(t, s) Q_{2}(s) x=\left(q_{n}(t, s)\right)_{n \geq 0}$, with

$$
\begin{aligned}
q_{4 n}(t, s) & =0 \\
q_{4 n+1}(t, s) & =\frac{t+1}{s+1} x_{4 n+1}=0 \\
q_{4 n+2}(t, s) & =0 \\
q_{4 n+3}(t, s) & =0
\end{aligned}
$$

it follows that $\Phi_{2}(t, s) Q_{2}(s) x=0$, which contradicts Remark 15; hence $\left(\Phi_{2}, P_{2}, Q_{2}, R_{2}\right)$ is not (s.p.t.).

Example 6 (trichotomy quadruple that is (w.p.t.) but fails to be (s.p.t.)). Let $\left(\Phi_{2}, P_{2}, Q_{2}, R_{2}\right)$ be the trichotomy quadruple from Example 5. Taking into account that, for all $s \geq 0$, $\left\|P_{2}(s)\right\|=\left\|Q_{2}(s)\right\|=\left\|R_{2}(s)\right\|=1$, it follows that $\left(\Phi_{2}, P_{2}, Q_{2}, R_{2}\right)$ is (u.w.p.t.) and hence (w.p.t.).

Again, from Example 5, we get that $\left(\Phi_{2}, P_{2}, Q_{2}, R_{2}\right)$ is not (s.p.t.) and hence not (u.s.p.t.).

\section{Conflict of Interests}

The authors declare that there is no conflict of interests regarding the publication of this paper.

\section{References}

[1] O. Perron, "Die stabilitätsfrage bei Differentialgleichungen," Mathematische Zeitschrift, vol. 32, no. 1, pp. 703-728, 1930.

[2] M.-G. Babuţia, T. Ceauşu, and N. M. Seimeanu, "On uniform exponential dichotomy of evolution operators," Annals of West University of Timisoara-Mathematics and Computer Science, vol. 50, no. 2, pp. 3-14, 2013.

[3] M.-G. Babuttia, M. Megan, and I.-L. Popa, "On $(h, k)$ dichotomies for nonautonomous linear difference equations in Banach spaces," International Journal of Differential Equations, vol. 2013, Article ID 761680, 7 pages, 2013.

[4] L. Barreira, M. Fan, C. Valls, and J. Zhang, "Robustness of nonuniform polynomial dichotomies for difference equations," Topological Methods in Nonlinear Analysis, vol. 37, no. 2, pp. 357376, 2011.

[5] L. Barreira and C. Valls, "Nonuniform exponential dichotomies and admissibility," Discrete and Continuous Dynamical Systems, vol. 30, no. 1, pp. 39-53, 2011.

[6] L. Barreira and C. Valls, "Polynomial growth rates," Nonlinear Analysis: Theory, Methods \& Applications, vol. 71, no. 11, pp. 5208-5219, 2009.

[7] N. Lupa and M. Megan, "Exponential dichotomies of evolution operators in Banach spaces," Monatshefte für Mathematik, vol. 174, no. 2, pp. 265-284, 2014.

[8] M. Megan, A. L. Sasu, and B. Sasu, "Discrete admissibility and exponential dichotomy for evolution families," Discrete and Continuous Dynamical Systems, vol. 9, no. 2, pp. 383-397, 2003.

[9] M. L. Rămneanțu, T. Ceauşu, and M. Megan, "On nonuniform polynomial dichotomy of evolution operators in Banach spaces," International Journal of Pure and Applied Mathematics, vol. 75, no. 3, pp. 305-318, 2012. 
[10] A. L. Sasu, M.-G. Babuţia, and B. Sasu, "Admissibility and nonuniform exponential dichotomy on the half-line," Bulletin des Sciences Mathematiques, vol. 137, no. 4, pp. 466-484, 2013.

[11] A. L. Sasu and B. Sasu, "Discrete admissibility and exponential trichotomy of dynamical systems," Discrete and Continuous Dynamical Systems Series A, vol. 34, no. 7, pp. 2929-2962, 2014.

[12] R. J. Sacker and G. R. Sell, "Existence of dichotomies and invariant splittings for linear differential systems I," Journal of Differential Equations, vol. 15, no. 3, pp. 429-458, 1974.

[13] L. Barreira and C. Valls, "Lyapunov functions for trichotomies with growth rates," Journal of Differential Equations, vol. 248, no. 1, pp. 151-183, 2010.

[14] L. Barreira and C. Valls, "Robustness of nonuniform exponential trichotomies in Banach spaces," Journal of Mathematical Analysis and Applications, vol. 351, no. 1, pp. 373-381, 2009.

[15] N. Lupa and M. Megan, "Generalized exponential trichotomies for abstract evolution operators on the real line," Journal of Function Spaces and Applications, vol. 2013, Article ID 409049, 8 pages, 2013.

[16] M. Megan and C. Stoica, "On uniform exponential trichotomy of evolution operators in Banach spaces," Integral Equations and Operator Theory, vol. 60, no. 4, pp. 499-506, 2008.

[17] M. Megan and C. Stoica, "Trichotomy for discrete skewevolution semiows in Banach spaces," Annals of the Tiberiu Popoviciu Seminar of Functional Equations, Approximation and Convexity, vol. 5, pp. 79-85, 2007.

[18] A. L. Sasu and B. Sasu, "Exponential trichotomy for variational difference equations," Journal of Difference Equations and Applications, vol. 15, no. 7, pp. 693-718, 2009. 


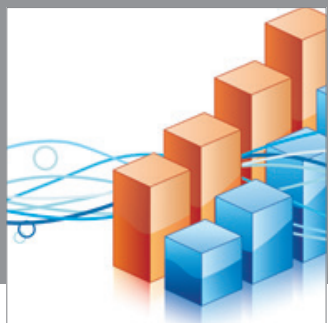

Advances in

Operations Research

mansans

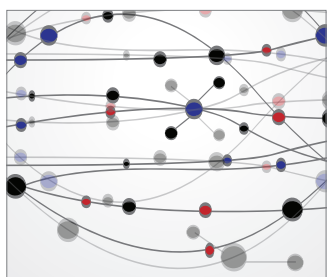

The Scientific World Journal
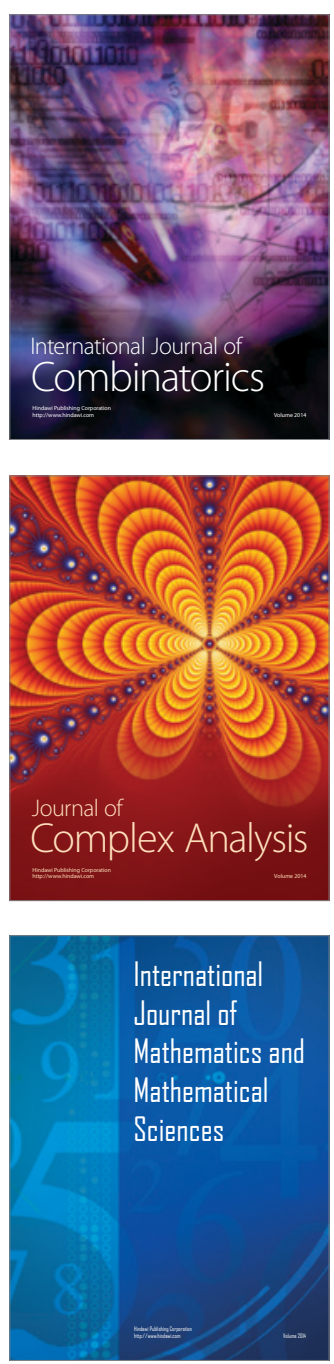
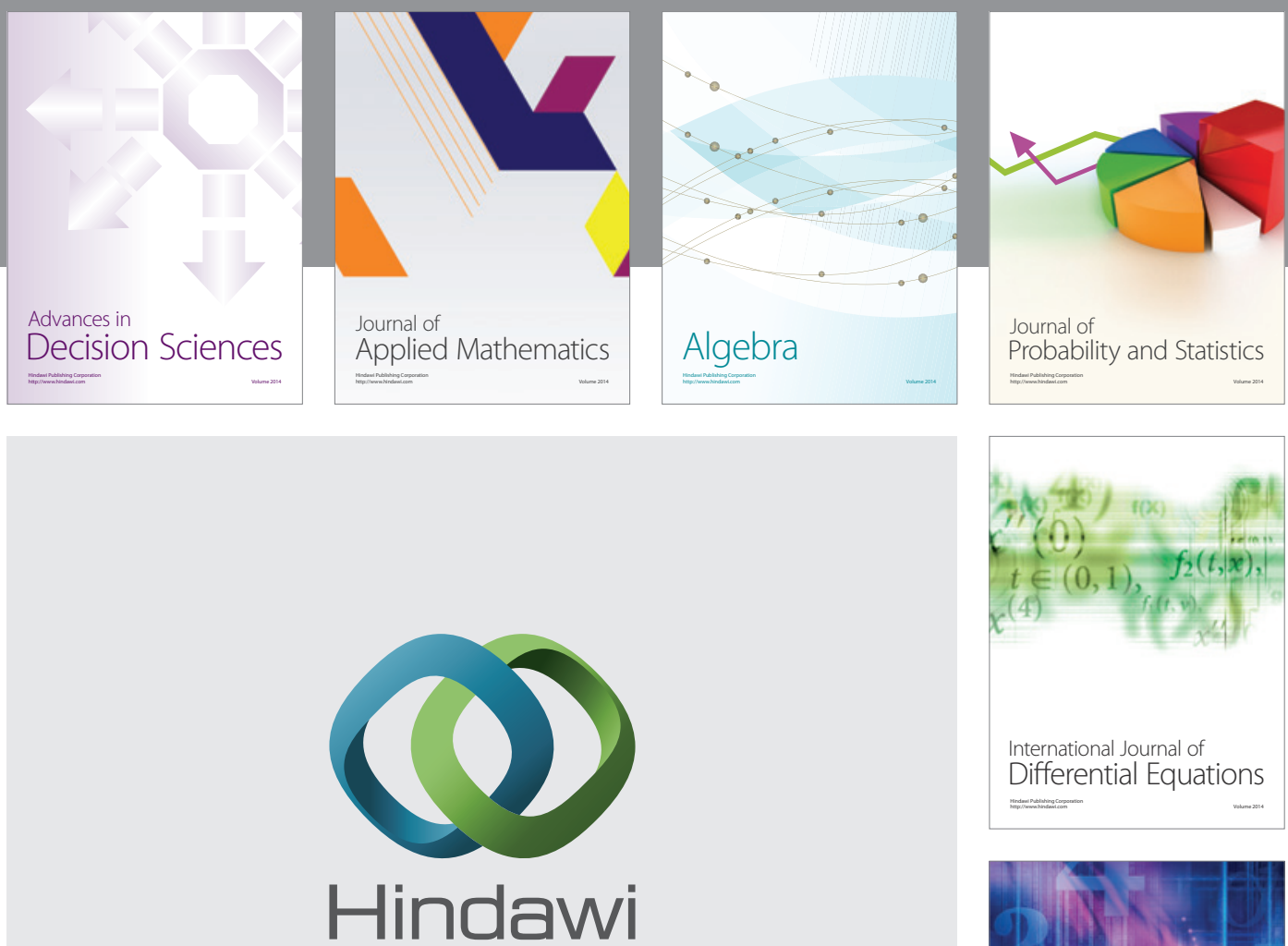

Submit your manuscripts at http://www.hindawi.com
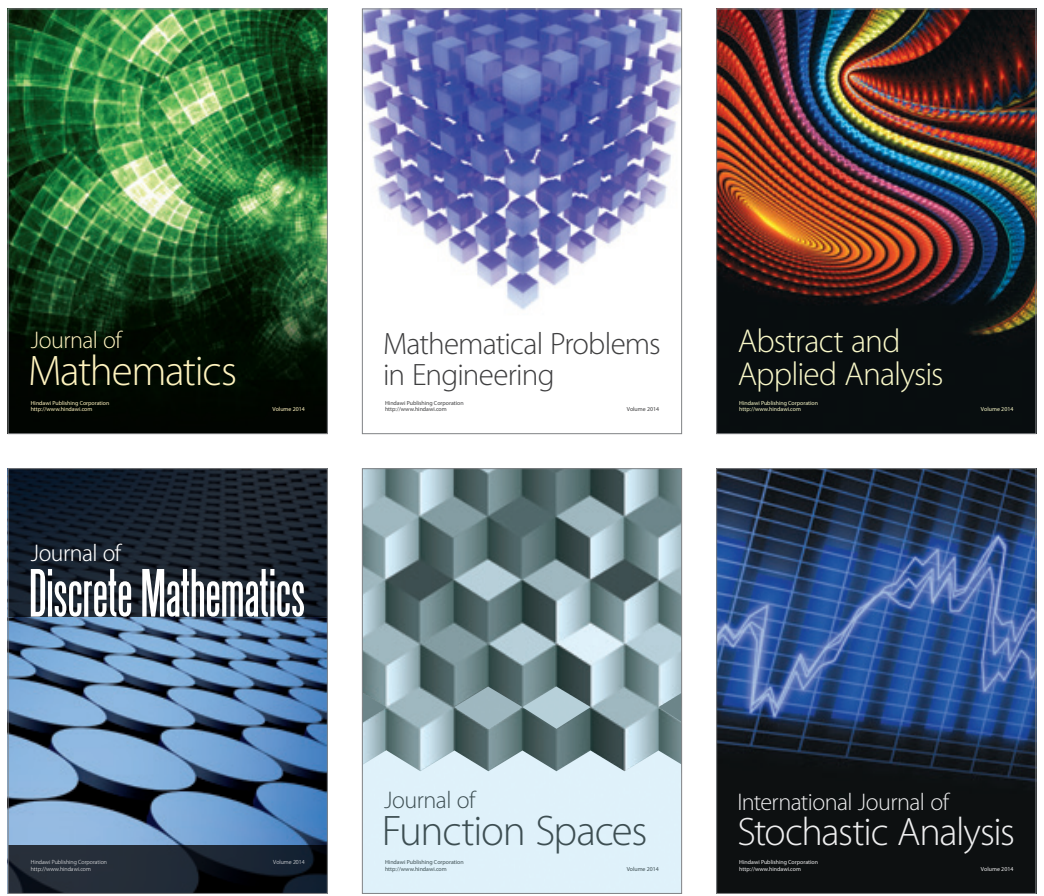

Journal of

Function Spaces

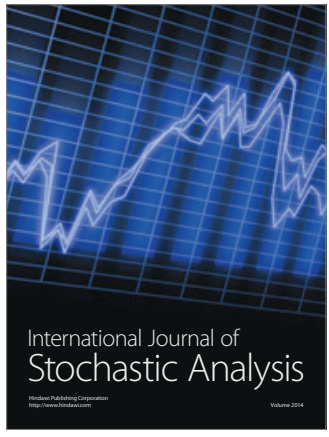

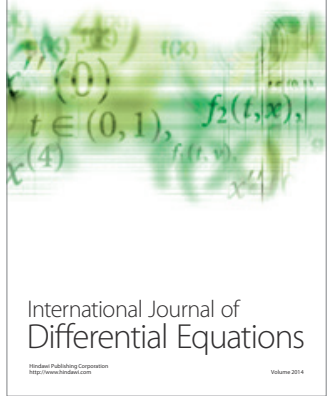
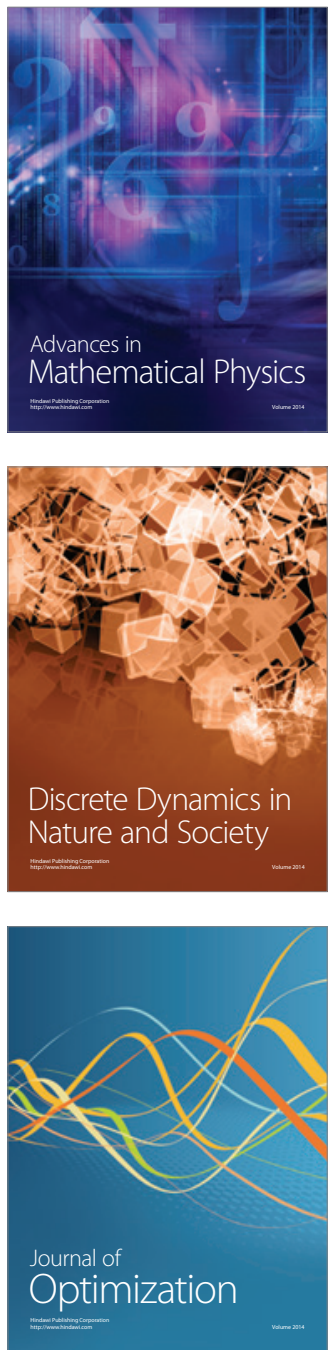